\title{
CD23 expression in mantle cell lymphoma is associated with CD200 expression, leukemic non-nodal form and a better prognosis
}

\author{
Annapurna Saksena MD ${ }^{1,2}$, C. Cameron Yin MD, Ph.D ${ }^{1}$, Jie Xu MD Ph.D ${ }^{1}$, Jingyi Li MD ${ }^{1,3}$, \\ Jiehao Zhou MD, Ph.D ${ }^{4}$, Sa A. Wang MD ${ }^{1}$, Pei Lin MD ${ }^{1}$, Guilin Tang MD, Ph.D ${ }^{1}$, Lifu Wang \\ $\mathrm{MD}^{1,5}$, Michael Wang MD ${ }^{6}$, Roberto N. Miranda MD ${ }^{1}$, L. Jeffrey Medeiros MD ${ }^{1}$, Shaoying Li \\ $\mathrm{MD}^{1 *}$ \\ ${ }^{1}$ Department of Hematopathology and ${ }^{6}$ Lymphoma and Myeloma, UT MD Anderson Cancer \\ Center, Houston, TX 77030, USA \\ ${ }^{2}$ Department of Pathology, UT Health San Antonio, TX 78229, USA \\ ${ }^{3}$ Department of Hematology/Oncology, Tianjin Hospital, Tianjin, China \\ ${ }^{4}$ Department of Pathology, Indiana University, Indianapolis, IN 46202, USA \\ ${ }^{5}$ Department of Pathology, Henan Provincial Hospital, Zhengzhou, Henan, China
}

\section{Declaration of interest: None}

Funding sources: None

Submission Declaration: Part of this research has been presented at the USCAP meeting

Running Title: CD23+ Mantle Cell Lymphoma

\section{*Corresponding author:}

Shaoying Li, M.D.

MD Anderson Cancer Center

Department of Hematopathology

1515 Holcombe Blvd. Unit 072

Houston, Texas 77030, USA

Phone: 713-792-2601

Fax: 713-563-3166

SLi6@mdanderson.org

This is the author's manuscript of the article published in final edited form as:

Saksena, A., Yin, C. C., Xu, J., Li, J., Zhou, J., Wang, S. A., ... Li, S. (2019). CD23 expression in mantle cell lymphoma is associated with CD200 expression, leukemic non-nodal form, and a better prognosis. Human Pathology, 89, 71-80. 


\begin{abstract}
Mantle cell lymphoma (MCL) is usually CD23-negative, a feature helpful in distinguishing MCL from chronic lymphocytic leukemia/small lymphocytic lymphoma (CLL/SLL). However, a subset of MCL cases can be CD23+. Limited data are available regarding the clinicopathologic features and prognosis of patients with CD23+ MCL. In this study, we reviewed 798 cases of MCL and identified 103 (13\%) that were CD23+ by flow cytometry, all of which were positive for cyclin D1 and/or associated with CCND1/IGH. In all cases of CD23+ MCL, CD23 expression was dim partial or dim, unlike moderate to bright CD23 expression observed in CLL/SLL. The clinicopathologic features and outcome of patients with CD23+ MCL were compared with 240 patients with typical MCL negative for CD23. Patients with CD23+ MCL more often had an elevated leukocyte count ( $33 \%$ vs $18 \%, \mathrm{p}=0.009)$, bone marrow involvement ( $89 \%$ vs $78 \%, p=0.02)$, stage 4 disease $(87 \%$ vs $77 \%, p=0.03$ ) and a leukemic presentation ( $42 \%$ vs $11 \%, \mathrm{p}=0.0001$ ). CD23+ MCL was also more often positive for CD200 (17\% vs. 4.6\%, $\mathrm{p}=0.0005)$ and less commonly positive for SOX11 (55\% vs. 74\%, p=0.027). All other clinicopathologic features were similar. With similar treatment regimens and observation times, patients with CD23+ MCL had a significant better overall survival $(\mathrm{p}=0.02)$ and progression-free survival ( $\mathrm{p}=0.029)$. In conclusion, CD23 expression was observed in 13\% of MCL cases and is associated with a better prognosis in patients with MCL. CD23 is associated with leukocytosis, a leukemic presentation, bone marrow involvement, CD200 expression, and a lower frequency of SOX11 positivity.
\end{abstract}

Key Words: CD23, mantle cell lymphoma, CD200, prognosis 


\section{INTRODUCTION}

Mantle cell lymphoma (MCL) is a distinctive type of B-cell lymphoma that represents 4-9\% of all cases of non-Hodgkin lymphoma depending on geographic region.[1] The genetic hallmark of MCL is $\mathrm{t}(11 ; 14)(\mathrm{q} 13 ; \mathrm{q} 32)$ which juxtaposes $C C N D 1$ on chromosome 11q13 with IGH on chromosome 14q32, resulting in overexpression of cyclin D1. Over-expression of cyclin D1 leads to cell cycle dysregulation and uncontrolled lymphoma cell growth. Patients with MCL have a poorer prognosis than patients with other types of small B-cell lymphoma; the median overall survival of MCL patients reported in the literature is $3-5$ years. The treatment regimens for MCL are therefore more intensive, unlike the therapies for other small B-cell lymphomas.[2] Nevertheless, as currently defined, MCL is heterogeneous with variable morphologic, immunophenotypic, cytogenetic, and molecular features as well as outcomes.[3-6] Recently, leukemic non-nodal forms of MCL that are clinically indolent have been reported in a small subset of patients [2, 4-9]. Patients with leukemic non-nodal MCL present with peripheral blood, bone marrow, and/or splenic involvement, but lack significant lymphadenopathy, the latter defined as lymph nodes $<1-2 \mathrm{~cm}$ by imaging studies.[10]

Mantle cell lymphoma has a distinctive immunophenotype, typically positive for pan B-cell markers, CD5 and cyclin D1, but negative for CD10, CD23, and CD200. Although most cases show this immunophenotype, some MCL cases have atypical immunophenotypic features, such as expression of CD10, CD23, or rarely CD200 or lack of expression of CD5. Earlier studies in the literature have shown that CD23 expression is the most common variant immunophenotypic finding in MCL.

CD23 is a transmembrane molecule that functions as a low-affinity IgE Fc receptor. CD23 is present in a wide variety of cell types including B cells, activated $\mathrm{T}$ cells, macrophages, eosinophils, platelets, follicular dendritic cells, and natural killer cells.[11] Schuurman et al first described CD23 as a marker for distinguishing MCL from CLL using frozen section immunohistochemical analysis.[12] Subsequent studies performed using blood smears, fresh or frozen or paraffin-embedded tissue specimens, and immunohistochemical analysis and flow cytometry immunophenotypic analysis also showed a significant difference in CD23 expression between MCL and CLL/SLL.[13-20] It was therefore generally accepted that CD23 is expressed in CLL/SLL, but is usually negative in MCL.[16] However, subsequent studies, mainly using 
flow cytometry methods with improved sensitivity, have shown that a small subset of MCL cases also express CD23.[16, 21, 22] Although a few studies on CD23 expression in MCL have been reported, most studies have reported a small number of cases and data regarding the clinicopathologic features and prognosis of patients with CD23+ MCL are limited.

In this retrospective study, we review a large series of CD23+ MCL cases and we assess the clinicopathologic features, immunophenotype, and prognosis of patients with CD23+ MCL.

\section{MATERIALS AND METHODS}

\subsection{Case Selection}

We searched the database of the Department of Hematopathology at The University of Texas MD Anderson Cancer Center from 2008 to 2016 for cases of MCL that were positive for CD23. The diagnosis of MCL was based on morphologic and immunophenotypic criteria as specified in the World Health Organization (WHO) classification. Specifically all cases had cyclin D1 expression by immunohistochemistry (IHC) and/or evidence of $\mathrm{t}(11 ; 14)(\mathrm{q} 13 ; \mathrm{q} 32) / C C N D 1-I G H$ or variant translocation confirmed by fluorescence in situ hybridization (FISH) or conventional karyotype analysis. Clinical information was obtained by review of the medical records. The clinicopathologic and immunophenotypic, features, overall survival (OS), and progression-free survival (PFS) of patients with CD23+ MCL were compared with 240 randomly chosen, wellcharacterized CD23-negative MCL cases, which were from the same time period with a similar distribution over the years as the CD23+ MCL group. This study included 17 cases that were reported previously in an independently performed study of CD200 expression in MCL. ${ }^{19}$ The study was approved by the institutional review board.

\subsection{Immunophenotypic Studies}

All cases were analyzed with FACSCalibur ${ }^{\mathrm{TM}}$ cytometers (BD Biosciences, San Jose, CA) before 2009, and with Canto II 8-color instruments (BD Biosciences) after 2009 as has been described.[23, 24] Briefly, the panel of antibodies used was variable over the years, but usually 
included CD3, CD5, CD10, FMC7, CD19, CD20, CD23, CD38, CD43, CD200 and surface immunoglobulin kappa and lambda light chains. All antibodies were obtained from BectonDickinson Biosciences. Lymphocytes were gated for analysis using CD45 expression and side scatter. CD23 expression were assessed on CD5+/CD19+ aberrant B cell population, with T cells as internal negative control and also fluorescence minus one as control. CD23 expression was defined as positive when at least $20 \%$ of aberrant B cells had expression, an arbitrary cutoff used to define positive expression in most of the biomarker studies by flow cytometry analysis.

Immunohistochemical studies were performed using formalin-fixed, paraffin-embedded tissue sections, either at the time of diagnosis or retrospectively for this study. After deparaffinization and dehydration of sections in graded alcohols and xylene, endogenous peroxidase was blocked with hydrogen peroxide. Heat-induced epitope retrieval was performed using citrate buffer, $\mathrm{pH}$ 6.0. The panel of monoclonal antibodies used was variable over time, but included reagents specific for CD3 polyclonal, DAKO, Carpinteria, CA, USA), CD5 (Novocastra, Newcastle-onTyne, UK), CD10 (DAKO), CD20 (DAKO), CD23 (Zymed, South San Francisco, CA, USA), BCL2 (clone 124, DAKO), BCL6 (DAKO), Ki-67 (DAKO); SOX-11(Cell Marque, Rocklin, California, USA) and cyclin D1 (clone AM29, NeoMarkers, Fremont, CA). The cutoff for positive SOX11 expression was defined as expression in $\geq 10 \%$ of cells as reported previously.[25]

\subsection{Cytogenetic Studies}

Conventional G-band karyotype analysis was performed on 24 cases using cell suspensions of lymph node, extranodal tissue specimens, or bone marrow aspirate specimens according to standard laboratory procedures as have been described.[26] The karyotypes were reported according to the 2016 International System for Human Cytogenetic Nomenclature.[27]

Fluorescence in situ hybridization (FISH) analysis was performed on 63 cases using a dual color and dual fusion probe set for CCND1/IGH (Abbott Molecular, Downers Grove, IL, USA). For 
bone marrow aspirate specimens, FISH was performed by using a freshly dropped slide from a harvested bone marrow aspirate specimen or a G-banded slide for metaphase mapping according to the manufacturer's instructions. For formalin-fixed, paraffin-embedded tissue samples, FISH was performed on 4-mm tissue sections and fixed onto slides as per the manufacturer's protocols. At least 200 interphase nuclei were analyzed and the sample was considered positive for $C C N D 1$ rearrangement if more than $2 \%$ of nuclei showed positive fusion signals.

\subsection{Statistical Analysis}

The Fisher exact test was utilized to analyze differences between the CD23+ and CD23-negative groups. OS was calculated from the date of diagnosis to the date of death or last follow-up. PFS was calculated from the date of diagnosis to the date of progression/relapse or, if no progression/relapse, the date of death or last follow-up. Patient survival was analyzed using the Kaplan-Meier method and compared using the log-rank test. Univariate and multivariate Cox proportional hazards model analyses for OS and PFS were performed by SPSS 22.0 software). A $P$-value of less than 0.05 was considered statistically significant.

\section{RESULTS}

\subsection{Clinical Characteristics}

From a total of 798 MCL cases evaluated by flow cytometry immunophenotypic analysis, 103 (13\%) were CD23+ and formed the study group. There were 81 men and 22 women with a median age of 65 years (range, 44 to 88 years) at the time of diagnosis of MCL (Table I). Sixtyseven $(65 \%)$ patients were older than 60 years old and there was a male predominance. Of 89 patients with a complete blood count (CBC) available at diagnosis, 29 (33\%) had a leucocyte count above the normal upper limit $\left(10 \times 10 \% / \mathrm{mm}^{3}\right)$. Twenty-three of $88(26 \%)$ patients had an elevated serum lactate dehydrogenase (LDH) level, 90 (87\%) patients had an advanced Ann Arbor stage (stage IV), and 15 (18\%) patients had a high MCL international prognostic index (MIPI) score [28, 29]. Bone marrow (BM) involvement was detected in 90 of $101(89 \%)$ patients and $43(42 \%)$ patients had a leukemic non-nodal presentation. 
CD23+ MCL patients were younger than CD23-negative MCL patients (median age 63 vs. 67 years, $\mathrm{p}=0.02$ ) (Table I). Patients with CD23+ MCL also more commonly had a leukemic nonnodal presentation ( $42 \%$ vs $11 \%, \mathrm{P}=0.0001)$ and elevated leukocyte count $(\mathrm{P}=0.009)$, largely due to significantly increased circulating lymphoma cells $(\mathrm{P}=0.03)$. Stage 4 disease with bone marrow involvement was more common in the CD23+ MCL patients, mostly attributable to the leukemic non-nodal presentation. The MIPI score and serum LDH level were similar between the two groups.

Among the 103 cases of CD23+ MCL, 53 cases had been assessed for SOX11 expression by immunohistochemistry and 29 cases were positive. The clinical features were further compared between patients with CD23+/SOX11+ MCL and those with CD23+/SOX11-negative MCL. Most features were similar between these subgroups $(\mathrm{p}>0.05)$ except that more patients were under observation in the CD23+/SOX11-negative group (Table II; 36\% in SOX11-negative vs $11 \%$ in SOX11+ group).

Among the 103 cases of CD23+ MCL, 43 cases were leukemic non-nodal (LNN) form and 60 were non-LNN. The clinical features were further compared between these two sub-groups. Patients with LNN disease had a significantly higher white blood cell count; more frequently had a high MIPI; more commonly had bone marrow involvement and therefore stage 4 disease; more frequent CD200 expression, and less frequently had a high Ki67 proliferation rate (Table III, $\mathrm{p}<0.05)$. Other features were similar between these groups.

\subsection{Morphologic and Immunophenotypic findings}

83 of $103(81 \%)$ CD23+ MCL cases had classical morphology (Figure 1) and the remaining 20 (19\%) cases had blastoid or pleomorphic morphology (Table I). All 96 cases tested for cyclin D1 by immunohistochemistry (Figure 1) were positive. The 7 cases not tested for cyclin D1 were positive for CCND1 rearrangement by FISH. All MCL cases showed moderate to bright CD20 
expression (Figure 1) and at least moderate light chain expression, except one case with dim kappa light chain. 97 (94\%) of CD23+ MCL cases were positive for CD5 and 2 (1.9\%) cases were positive for CD10. SOX11 was assessed by immunohistochemistry in 53 cases and was positive in $29(55 \%)$.

CD23 expression was detected by flow cytometry in all cases; $69(67 \%)$ cases exhibited partial dim CD23 expression and 34 (33\%) cases had dim or occasionally dim to moderate expression. No cases strongly expressed CD23. (Figure 1). Fifteen CD23+ MCL cases were also assessed for CD23 by immunohistochemistry. Six of 13 MCL cases with dim partial CD23 expression were positive by immunohistochemistry and 7 cases were negative. Two cases of MCL with uniform $\operatorname{dim}$ CD23 expression were also positive by immunohistochemistry.

All MCL cases were assessed for CD200 expression by flow cytometry which was positive in 17 (16.5\%) cases; 13 cases had dim partial CD200 and 4 had uniform CD200 expression. Twelve of 17 CD23+/CD200+ MCL were assessed for SOX11 and all were negative. These cases were further tested for LEF1 expression by immunohistochemistry and all were negative. The Ki-67 proliferation rate in CD23+ MCL was highly variable, ranging from 5\% to 95\%, with 26 of 60 $(43 \%)$ cases having a proliferation rate $>30 \%$.

There was no significant difference in the frequency of morphologic subtypes between CD23+ and CD23-negative MCL. CD200 expression was significantly more common in CD23+ MCL than in typical CD23-negative MCL (16.5\% vs 4.6\%, p=0.0005), and in leukemic non-nodal cases than other cases within the CD23+ group. In contrast, SOX11 expression was significantly less common in CD23+ MCL cases ( $55 \%$ vs $74 \%, \mathrm{p}=0.027$, Table I).

\subsection{Treatment and Response}

Detailed treatment information was available in 99 patients. Thirty-six (36\%) patients were treated with aggressive induction therapy, mainly R-Hyper CVAD therapy (rituximab, 
fractionated cyclophosphamide, vincristine, doxorubicin, and dexamethasone alternating with high dose methotrexate and cytarabine). Sixteen (16\%) patients were observed without treatment at last follow up and 47 patients received other treatment regimens. After induction, 63 patients achieved complete remission (CR) and 32 (32\%) patients relapsed, progressed or primary refractory. Twelve patients also underwent stem cell transplantation.

The frequency of patients who received aggressive versus less aggressive induction chemotherapy regimens and the frequency of CR rate were not significantly different between the CD23+ and CD23-negative MCL groups ( $>0.05$ ). However, patients with CD23+ MCL had lower rates of relapse/progression or refractory disease were than patients with CD23-negative MCL (32\% vs $44 \%, \mathrm{p}=0.048)$.

\subsection{Prognosis}

With a median follow-up of 37 months (range 1.6 - 124 months), 16 (16\%) of 103 patients had died at the time of last follow-up. The 5-year overall survival rate was $84 \%$ and the progressionfree survival rate was 59\%, significantly better than for patients with CD23-negative MCL (64\% and $39 \%$ respectively, $\mathrm{p}<0.05$; Figure $2 \mathrm{~A} \& \mathrm{~B}$ ). To examine if this survival advantage is related to the inclusion of SOX11-negative cases, the OS and PFS were compared between patients with CD23+/SOX11+ MCL versus those with CD23+/SOX11-negative MCL and there was no significant difference in both OS and PFS ( $p>0.05$; Figure 2 C\&D). Survival was also compared between patients with leukemic non-nodal disease (LNN) and those with non-LNN MCL. Patients with a LNN presentation showed a trend towards better OS ( $p=0.12$, Figure $2 \mathrm{E})$. There was also no significant difference in PFS ( $\mathrm{p}=0.58$; Figure $2 \mathrm{~F}$ )

The prognostic impact of age, leukocyte count, morphology, Ki-67, and MIPI score were examined in CD23+ MCL patients. Blastoid/pleomorphic morphology, Ki-67 >30\%, and high MIPI score were associated with a worse OS ( $<<0.05$, Figure 3 A-C). Both blastoid/pleomorphic morphology and high MIPI were associated with a poorer PFS in patients with CD23+ MCL $(p=$ 0.002 and 0.001, respectively; Figure 3 D \& F). Ki-67 was not associated with PFS (p=0.33; Figure 3 E). Age and leukocyte count did not predict OS or PFS in patients with CD23+ MCL. 
As LNN disease has been associated with a better OS and a tendency towards better PFS when all cases of MCL were included (Figure $4 \mathrm{~A} \& \mathrm{~B}, \mathrm{P}=0.009$ and 0.128 respectively), OS was further compared between CD23+ and CD23-negative groups when patients with LNN disease were excluded. As shown in Figures $4 \mathrm{C}$ and D, CD23 expression was not associated with OS, but there is still a trend towards better PFS when patients with LNN disease were excluded $(\mathrm{P}=0.216$ and 0.066 respectively)

Multivariate Cox regression analysis was performed to identify independent prognostic factors for OS and PFS in all MCL patients, including CD23+ MCL as well as typical CD23-negative MCL (Table IV). Ki-67 >30\% and high MIPI were significantly associated with shorter OS $(\mathrm{P}=0.00)$. High MIPI was also significantly associated with shorter PFS $(\mathrm{p}=0.00) . \mathrm{CD} 23$ expression did not predict survival in multivariate analysis 


\section{DISCUSSION}

Historically, CD23 expression was reported to be absent or rare in MCL, but positive in CLL/SLL, making this marker useful in distinguishing these two neoplasms (1). However, using the more sensitive technique of flow cytometry immunophenotypic analysis, the frequency of CD23+ MCL in several small series studies has varied from $0 \%$ to $45 \%$ [15-17, 20-22]. In this large retrospective study, we assessed for CD23 in 798 cases of MCL and 103 (13\%) cases were CD23+. In almost all cases, CD23 was expressed dimly and, in about two thirds of cases, CD23 expression was also partial. Only occasional cases had dim-moderate intensity expression and no cases were strongly for CD23+.

In this cohort, patients with CD23+ MCL had a lower median age and lower frequency of patients older than 60 years that in CD23-negative MCL patients. Patients with CD23+ MCL also more frequently had stage 4 disease with bone marrow involvement as well as an elevated leukocyte count. Not surprisingly, a leukemic non-nodal (LNN) presentation was significantly more common in patients with CD23+ MCL than those with CD23-negative MCL (42\% versus $11 \%$ ). This result is in contrast to a study by Kelemen et al[21] who showed a similar frequency of bone marrow and peripheral blood involvement, but a higher frequency of extranodal nonmedullary disease in patients with CD23+ MCL compared with patients with CD23-negative MCL.

There are no studies in literature correlating CD23 and SOX11 expression in MCL. We found that CD23+ MCL is more often SOX11 negative than CD23-negative MCL ( $p=0.027$, Table 1). In addition, CD23 expression was significantly associated with CD200 expression, 17\% in CD23+ MCL group vs $4.6 \%$ in CD23-negative MCL. In an earlier, independently performed study from our department, $\mathrm{Hu}$ and colleagues reported that $76 \%$ of cases of CD200+ MCL express CD23.[23] With these correlations, we identified a small subgroup of MCL cases with CLL-like features, being associated commonly with a leukemic presentation and with an immunophenotype similar to CLL/SLL, being CD23+, CD200+, and SOX11-negative. CD23 expression is often used as a marker to distinguish MCL from CLL/SLL. However, cases of 
CD23+ MCL with CLL-like features are diagnostically challenging. Although there are well established morphological criteria, MCL may show a variety of histologic and cytologic patterns, some of which can mimic CLL/SLL. It can be especially difficult to distinguish MCL from CLL/SLL in peripheral blood or bone marrow.[30-32] For some of these MCL cases with CLLlike features, it can be challenging (if not impossible) to distinguish them from CLL/SLL by morphology and flow cytometry, even including SOX11 immunohistochemistry. In such cases, immunohistochemistry for cyclin D1 and FISH or karyotype to identify CCNDI/IGH or $\mathrm{t}(11 ; 14)(\mathrm{q} 13 ; \mathrm{q} 32)$ are the diagnostic tests. To avoid misdiagnosing these cases as CLL/SLL, an immunohistochemical study for cyclin D1 should be performed on new cases of CLL/SLL.

There are limited data available in the literature regarding the prognostic significance of CD23 expression in MCL. Kelemen et al [21] studied 14 patients of CD23+ MCL and compared this group to 33 patients with CD23-negative MCL and found that CD23 expression was associated with improved event-free survival $(\mathrm{p}=0.02)$ and possibly OS $(\mathrm{p}=0.07)$. However, CD23 was not an independent prognostic factor by multivariate analysis. In a study of 372 patients with CD5+ B cell lymphoproliferative disorders evaluated by flow cytometry immunophenotyping, DiRaimondo et al[33] reported that CD23 expression was associated with improved survival; MCL cases were not separately analyzed in this study. Gong et al [16] did not detect a survival difference according to CD23 expression, although the number of cases in their report was small and follow-up was short. In this study, we compared 103 cases of CD23+ MCL to a group of 240 patients with typical CD23-negative MCL, by far the largest series of CD23+ MCL. We found that the median OS and PFS were significantly better in patients with CD23+ MCL than in patients with CD23-negative MCL ( $\mathrm{p}<0.05$ for both) by univariate analysis. However, prognostic significance was lost partially when LNN cases were excluded; suggesting that the prognostic effect is at least partially related to the more frequent LNN form in the CD23+ group. Importantly, this survival advantage does not appear to be entirely related to the inclusion of SOX11-negative cases because the survival of patients with CD23+/SOX11-negative MCL was similar to those with CD23+/SOX11+ MCL. CD23 expression was not an independent prognostic factor in multivariate analysis. 
In summary, in this study of a large cohort of MCL cases we observed CD23 expression in 13\%. CD23 expression is usually dim and partial in MCL, unlike the moderate to strong positivity often seen in CLL/SLL. CD23 expression in MCL correlates with a higher frequency of CD200 expression, lower frequency of SOX11 expression, and a higher frequency of leukemic nonnodal presentation. Since previous studies of CD23+ MCL in the literature had a limited number of patients, little data are available regarding prognostic factors in CD23+ MCL patients to date. Here we assessed prognostic factors and show that morphology and MIPI score predicted for OS and PFS and that Ki-67 >30\% was associated with a worse overall survival. CD23 expression also predicts a better OS and PFS in MCL patients by univariate analysis, although the evaluation is somewhat limted by the various treatments. Lastly, this is the first study in the literature that identified a small subgroup of MCL cases that were CD23+, CD200+, SOX11negative, and a leukemic presentation, features similar to CLL/SLL. These cases pose a diagnostic challenge and stress the importance of assessing for cyclin D1 expression and/or CCND1 rearrangement in any newly diagnosed CLL/SLL or other CD5+ small B cell leukemia and lymphoma. 


\section{REFERENCES}

[1] J.M. Vose, Mantle cell lymphoma: 2017 update on diagnosis, risk-stratification, and clinical management, Am J Hematol 92(8) (2017) 806-813.

[2] C.Y. Cheah, J.F. Seymour, M.L. Wang, Mantle Cell Lymphoma, J Clin Oncol 34(11) (2016) 1256-69.

[3] M. Dreyling, V. Amador, M. Callanan, et al., Update on the molecular pathogenesis and targeted approaches of mantle cell lymphoma: summary of the 12th annual conference of the European Mantle Cell Lymphoma Network, Leuk Lymphoma 56(4) (2015) 866-76.

[4] V. Fernandez, O. Salamero, B. Espinet, et al., Genomic and gene expression profiling defines indolent forms of mantle cell lymphoma, Cancer Res 70(4) (2010) 1408-18.

[5] P. Jares, D. Colomer, E. Campo, Molecular pathogenesis of mantle cell lymphoma, J Clin Invest 122(10) (2012) 3416-23.

[6] B. Sander, L. Quintanilla-Martinez, G. Ott, et al., Mantle cell lymphoma--a spectrum from indolent to aggressive disease, Virchows Arch 468(3) (2016) 245-57.

[7] E.D. Hsi, P. Martin, Indolent mantle cell lymphoma, Leuk Lymphoma 55(4) (2014) 761-7.

[8] S.L. Ondrejka, R. Lai, S.D. Smith, et al., Indolent mantle cell leukemia: a clinicopathological variant characterized by isolated lymphocytosis, interstitial bone marrow involvement, kappa light chain restriction, and good prognosis, Haematologica 96(8) (2011) 1121-7.

[9] J. Orchard, R. Garand, Z. Davis, et al., A subset of t(11;14) lymphoma with mantle cell features displays mutated IgVH genes and includes patients with good prognosis, nonnodal disease, Blood 101(12) (2003) 4975-81.

[10] C.E. Swerdlow S. H., Seto M, and Muller-Hermelink H.K., Mantle cell lymphoma, in: C.E. Swerdlow S. H., Harris N.L, et al (Ed.), WHO Classification of Tumors of Hematopoietic and Lymphoid Tissues, IARC, Lyon, France, 2017.

[11] J. Gordon, L. Flores-Romo, J.A. Cairns, et al., CD23: a multi-functional receptor/lymphokine?, Immunology today 10(5) (1989) 153-7.

[12] H.J. Schuurman, J. van Baarlen, W. Huppes, et al., Immunophenotyping of non-Hodgkin's lymphoma. Lack of correlation between immunophenotype and cell morphology, The American journal of pathology 129(1) (1987) 140-51.

[13] E.D. de Leon, S. Alkan, J.C. Huang, et al., Usefulness of an immunohistochemical panel in paraffinembedded tissues for the differentiation of B-cell non-Hodgkin's lymphomas of small lymphocytes, Mod Pathol 11(11) (1998) 1046-51.

[14] D.M. Dorfman, G.S. Pinkus, Distinction between small lymphocytic and mantle cell lymphoma by immunoreactivity for CD23, Mod Pathol 7(3) (1994) 326-31.

[15] D.P. Garcia, M.T. Rooney, E. Ahmad, et al., Diagnostic usefulness of CD23 and FMC-7 antigen expression patterns in B-cell lymphoma classification, Am J Clin Pathol 115(2) (2001) 258-65.

[16] J.Z. Gong, A.S. Lagoo, D. Peters, et al., Value of CD23 determination by flow cytometry in differentiating mantle cell lymphoma from chronic lymphocytic leukemia/small lymphocytic lymphoma, Am J Clin Pathol 116(6) (2001) 893-7.

[17] M.N. Kilo, D.M. Dorfman, The utility of flow cytometric immunophenotypic analysis in the distinction of small lymphocytic lymphoma/chronic lymphocytic leukemia from mantle cell lymphoma, Am J Clin Pathol 105(4) (1996) 451-7.

[18] A. Orazi, G. Cattoretti, N. Polli, et al., Distinct morphophenotypic features of chronic B-cell leukaemias identified with CD1c and CD23 antibodies, Eur J Haematol 47(1) (1991) 28-35.

[19] N. Singh, D.H. Wright, The value of immunohistochemistry on paraffin wax embedded tissue sections in the differentiation of small lymphocytic and mantle cell lymphomas, J Clin Pathol 50(1) (1997) 16-21. 
[20] J.A. Tworek, T.P. Singleton, B. Schnitzer, et al., Flow cytometric and immunohistochemical analysis of small lymphocytic lymphoma, mantle cell lymphoma, and plasmacytoid small lymphocytic lymphoma, Am J Clin Pathol 110(5) (1998) 582-9.

[21] K. Kelemen, L.C. Peterson, I. Helenowski, et al., CD23+ mantle cell lymphoma: a clinical pathologic entity associated with superior outcome compared with CD23- disease, Am J Clin Pathol 130(2) (2008) 166-77.

[22] E. Schlette, K. Fu, L.J. Medeiros, CD23 expression in mantle cell lymphoma: clinicopathologic features of 18 cases, Am J Clin Pathol 120(5) (2003) 760-6.

[23] Z. Hu, Y. Sun, E.J. Schlette, et al., CD200 expression in mantle cell lymphoma identifies a unique subgroup of patients with frequent IGHV mutations, absence of SOX11 expression, and an indolent clinical course, Mod Pathol 31(2) (2018) 327-336.

[24] J. Xu, L.J. Medeiros, A. Saksena, et al., CD10-positive mantle cell lymphoma: clinicopathologic and prognostic study of 30 cases, Oncotarget 9(14) (2018) 11441-11450.

[25] I. Ribera-Cortada, D. Martinez, V. Amador, et al., Plasma cell and terminal B-cell differentiation in mantle cell lymphoma mainly occur in the SOX11-negative subtype, Mod Pathol 28(11) (2015) 1435-47.

[26] C.C. Yin, J. Peng, Y. Li, et al., Clinical significance of newly emerged isolated del(20q) in patients following cytotoxic therapies, Mod Pathol 28(8) (2015) 1014-22.

[27] S.M. Shaffer LG M-JJ, An International System for Human Cytogenetic Nomenclature (2013), 1st ed ed., Basel, Switzerland, 2012.

[28] E. Hoster, M. Dreyling, W. Klapper, et al., A new prognostic index (MIPI) for patients with advancedstage mantle cell lymphoma, Blood 111(2) (2008) 558-65.

[29] E. Hoster, W. Klapper, O. Hermine, et al., Confirmation of the mantle-cell lymphoma International Prognostic Index in randomized trials of the European Mantle-Cell Lymphoma Network, J Clin Oncol 32(13) (2014) 1338-46.

[30] P.L. Cohen, P.J. Kurtin, K.A. Donovan, et al., Bone marrow and peripheral blood involvement in mantle cell lymphoma, British journal of haematology 101(2) (1998) 302-10.

[31] J. Wasman, N.S. Rosenthal, D.C. Farhi, Mantle cell lymphoma. Morphologic findings in bone marrow involvement, American journal of clinical pathology 106(2) (1996) 196-200.

[32] K.F. Wong, J.K. Chan, J.C. So, et al., Mantle cell lymphoma in leukemic phase: characterization of its broad cytologic spectrum with emphasis on the importance of distinction from other chronic lymphoproliferative disorders, Cancer 86(5) (1999) 850-7.

[33] F. DiRaimondo, M. Albitar, Y. Huh, et al., The clinical and diagnostic relevance of CD23 expression in the chronic lymphoproliferative disease, Cancer 94(6) (2002) 1721-30. 


\section{Figure Legends}

Figure 1. A representative case of CD23+ mantle cell lymphoma. The lymphoma cells grew in a diffuse pattern (H\&E, 400X) and were CD20+, Cyclin D1+, SOX11+, and CD23+ (Immunohistochemistry, 400X). Flow cytometry study showed a kappa-restricted monoclonal B cell population that was positive for CD5, CD19, and CD23 (partial).

Figure 2. CD23 expression was associated with improved overall survival (OS) (A) and progression free survival (PFS) (B) in all MCL patients. But within the CD23+ MCL group, SOX11 expression or leukemic non-nodal disease are not associated with OS (C \& E) and PFS (D \& F).

Figure 3. Prognostic factors might be associated with overall survival (OS) and progression free survival (PFS) in CD23+ MCL: blastoid/pleomorphic type (A \& D), Ki-67 using 30\% as cutoff value (B \& E), high MIPI (C \& F).

Figure 4. Leukemic non-nodal disease is associated with better overall survival (OS, A) but not progression free survival (PFS, B) in all MCL. When these leukemic non-nodal cases are excluded, CD23 expression is not associated with OS (C), but has a tendency towards a better PFS (D). 
Table I: Clinical features of patients with CD23+ vs typical CD23-negative MCL

\begin{tabular}{lccc}
\hline & $\begin{array}{c}\text { CD23+ MCL (n=103) } \\
\text { \%(Positive/Evaluated) }\end{array}$ & $\begin{array}{c}\text { CD23-Negative MCL (n=240) } \\
\text { \%(Positive/Evaluated) }\end{array}$ & $\boldsymbol{P}$ \\
\hline Medium age (yrs, range) & $63(44-88)$ & $67(29-95)$ & $0.018^{\mathrm{a}}$ \\
Age $\geq$ 60 (yrs) & $65(67 / 103)$ & $76(182 / 240)$ & $0.048^{\mathrm{a}}$ \\
Male:Female & $81 / 22$ & $199 / 41$ & 0.364 \\
BM+ & $89(90 / 101)$ & $78(178 / 228)$ & $0.021^{\mathrm{a}}$ \\
Stage IV & $87(90 / 103)$ & $77(180 / 234)$ & $0.027^{\mathrm{a}}$ \\
Elevated WBC & $33(29 / 89)$ & $18(36 / 200)$ & $0.009^{\mathrm{a}}$ \\
Elevated serum LDH & $26(23 / 88)$ & $27(52 / 193)$ & 1.000 \\
High MIPI & $18(15 / 85)$ & $24(38 / 160)$ & 0.329 \\
Leukemic Non-Nodal & $42(43 / 103)$ & $11(26 / 239)$ & $0.0001^{\mathrm{a}}$ \\
Presentation & $19(20 / 103)$ & $20(49 / 240)$ & 0.884 \\
Blastoid/pleomorphic & $17(17 / 103)$ & $4.6(11 / 240)$ & $0.001^{\mathrm{a}}$ \\
CD200+ & $55(29 / 53)$ & $74(64 / 87)$ & $0.027^{\mathrm{a}}$ \\
SOX11+ & $43(26 / 60)$ & $43(65 / 152)$ & 1.000 \\
Ki67 >30\% & $36(36 / 99)$ & & 0.122 \\
Initial chemo & $47(47 / 99)$ & $43(95 / 219)$ & \\
$\quad$ HCVAD+R & $16(16 / 99)$ & $11(24 / 219)$ & \\
$\quad$ Other & Observation & & \\
\hline
\end{tabular}

a: all these parameters are statistically significantly different in the two groups. 
Table II. Clinical features of patients with CD23+ MCL with or without SOX11 expression

\begin{tabular}{|c|c|c|c|}
\hline & $\begin{array}{c}\text { CD23+/SOX11+ MCL } \\
\text { (n=29) } \\
\%(\text { Positive/Evaluated) }\end{array}$ & $\begin{array}{c}\text { CD23+/SOX11-negative MCL } \\
(\mathrm{n}=24) \\
\%(\text { Positive/Evaluated) }\end{array}$ & $\boldsymbol{P}$ \\
\hline Medium age (yrs, range) & $67(46-88)$ & $65(44-80)$ & 0.371 \\
\hline Age $\geq 60$ (yrs) & $79(23 / 29)$ & $63(15 / 24)$ & 0.227 \\
\hline Male:Female & $21 / 8$ & & 0.750 \\
\hline BM+ & $86(25 / 29)$ & $96(22 / 23)$ & 0.368 \\
\hline Stage IV & $86(25 / 29)$ & $96(23 / 24)$ & 0.362 \\
\hline Elevated WBC & $32(7 / 22)$ & $55(12 / 22)$ & 0.223 \\
\hline Elevated serum LDH & $27(6 / 22)$ & $19(4 / 21)$ & 0.721 \\
\hline High MIPI & $35(7 / 20)$ & $18(4 / 22)$ & 0.298 \\
\hline $\begin{array}{l}\text { Leukemic Non-Nodal } \\
\text { Presentation }\end{array}$ & $41(12 / 29)$ & $54(13 / 24)$ & 0.415 \\
\hline Blastoid/pleomorphic & $21(6 / 29)$ & $13(3 / 24)$ & 0.884 \\
\hline CD200+ & $0(0 / 29)$ & $50(12 / 24)$ & $0.000^{\mathrm{a}}$ \\
\hline Ki67 >30\% & $42(8 / 19)$ & $33(5 / 15)$ & 0.728 \\
\hline \multicolumn{4}{|l|}{ Initial chemo } \\
\hline HCVAD+R & 12/29) & $28(6 / 22)$ & \\
\hline Other & $48(14 / 29)$ & $36(8 / 22)$ & \\
\hline Observation & $11(3 / 29)$ & $36(8 / 22)$ & $0.039^{a}$ \\
\hline
\end{tabular}

a: all these parameters are statistically significantly different in the two groups. 
Table III. Clinical features of patients with leukemic non-nodal (LNN) vs Non-LNN CD23+ MCL

\begin{tabular}{|c|c|c|c|}
\hline & $\begin{array}{c}\text { CD23+/LNN MCL } \\
(n=43) \\
\%(P o s i t i v e / E v a l u a t e d)\end{array}$ & $\begin{array}{c}\text { CD23+/Non-LNN MCL }(n=60) \\
\%(\text { Positive/Evaluated) }\end{array}$ & $\boldsymbol{P}$ \\
\hline Medium age (yrs, range) & $64(46-88)$ & $63(44-83)$ & 0.247 \\
\hline Age $\geq 60$ (yrs) & $67(29 / 43)$ & $63(38 / 60)$ & 0.682 \\
\hline Male:Female & $35 / 8$ & $46 / 14$ & 0.632 \\
\hline $\mathbf{B M +}$ & $100(43 / 43)$ & $81(47 / 58)$ & $0.002^{\mathrm{a}}$ \\
\hline Stage IV & $100(43 / 43)$ & $82(49 / 60)$ & $0.002^{\mathrm{a}}$ \\
\hline Elevated WBC & $74(28 / 38)$ & $14(7 / 51)$ & $0.000^{\mathrm{a}}$ \\
\hline Elevated serum LDH & $24(9 / 37)$ & $27(14 / 51)$ & 0.809 \\
\hline High MIPI & $31(11 / 36)$ & $8(4 / 50)$ & $0.009^{a}$ \\
\hline Blastoid/pleomorphic & $5(2 / 43)$ & $13(3 / 24)$ & 0.341 \\
\hline CD200+ & $33(14 / 43)$ & $5(3 / 60)$ & $0.000^{a}$ \\
\hline Ki67 >30\% & $25(4)$ & $59(26 / 44)$ & $0.039^{a}$ \\
\hline \multicolumn{4}{|l|}{ Initial chemo } \\
\hline HCVAD+R & $29(12 / 42)$ & $40(23 / 57)$ & \\
\hline Other & $(19 / 42)$ & $49(28 / 57)$ & \\
\hline Observation & $26(11 / 42)$ & $11(6 / 57)$ & 0.288 \\
\hline
\end{tabular}


Table IV. Multivariate analysis in all MCL cases

\begin{tabular}{|c|c|c|c|c|c|c|}
\hline & \multicolumn{3}{|c|}{ Overall survival } & \multicolumn{3}{|c|}{ Progression free survival } \\
\hline & HR & $95 \% \mathrm{Cl}$ & $P$ & HR & $95 \% \mathrm{Cl}$ & $P$ \\
\hline CD23+ & 0.98 & $0.35-2.75$ & 0.96 & 0.84 & $0.40-1.77$ & 0.65 \\
\hline Ki67>30\% & 5.82 & $2.22-15.27$ & $0.00^{\mathrm{a}}$ & 1.79 & $0.86-3.70$ & 0.12 \\
\hline MIPI High & 3.69 & $1.70-8.05$ & $0.00^{a}$ & & $1.43-5.07$ & $0.00^{a}$ \\
\hline Blastoid/Pleumorphic & 0.76 & $0.32-1.82$ & 0.53 & 4 & $0.67-3.07$ & 0.35 \\
\hline $\begin{array}{l}\text { Leukemic Non-Nodal } \\
\text { Presentation }\end{array}$ & 0.40 & $0.08-2.11$ & 0.28 & 0.73 & $0.25-2.10$ & 0.56 \\
\hline
\end{tabular}

a: These parameters are statistically significantly different in the two groups. 


\section{CD23+ Mantle cell lymphoma (MCL) Highlights:}

- $\mathrm{CD} 23$ expression in MCL is dim/partial, in contrast to moderate/strong in CLL/SLL;

- $\mathrm{CD} 23$ expression is associated with better prognosis in patients with MCL;

- CD23 expression in MCL correlates with:

- A higher frequency of CD200 expression

- A lower frequency of SOX11 expression

- A higher frequency of leukemic non-nodal presentation

- A small subset of CD23+MCL has features similar to CLL (CD200+, SOX11-, leukemic) 


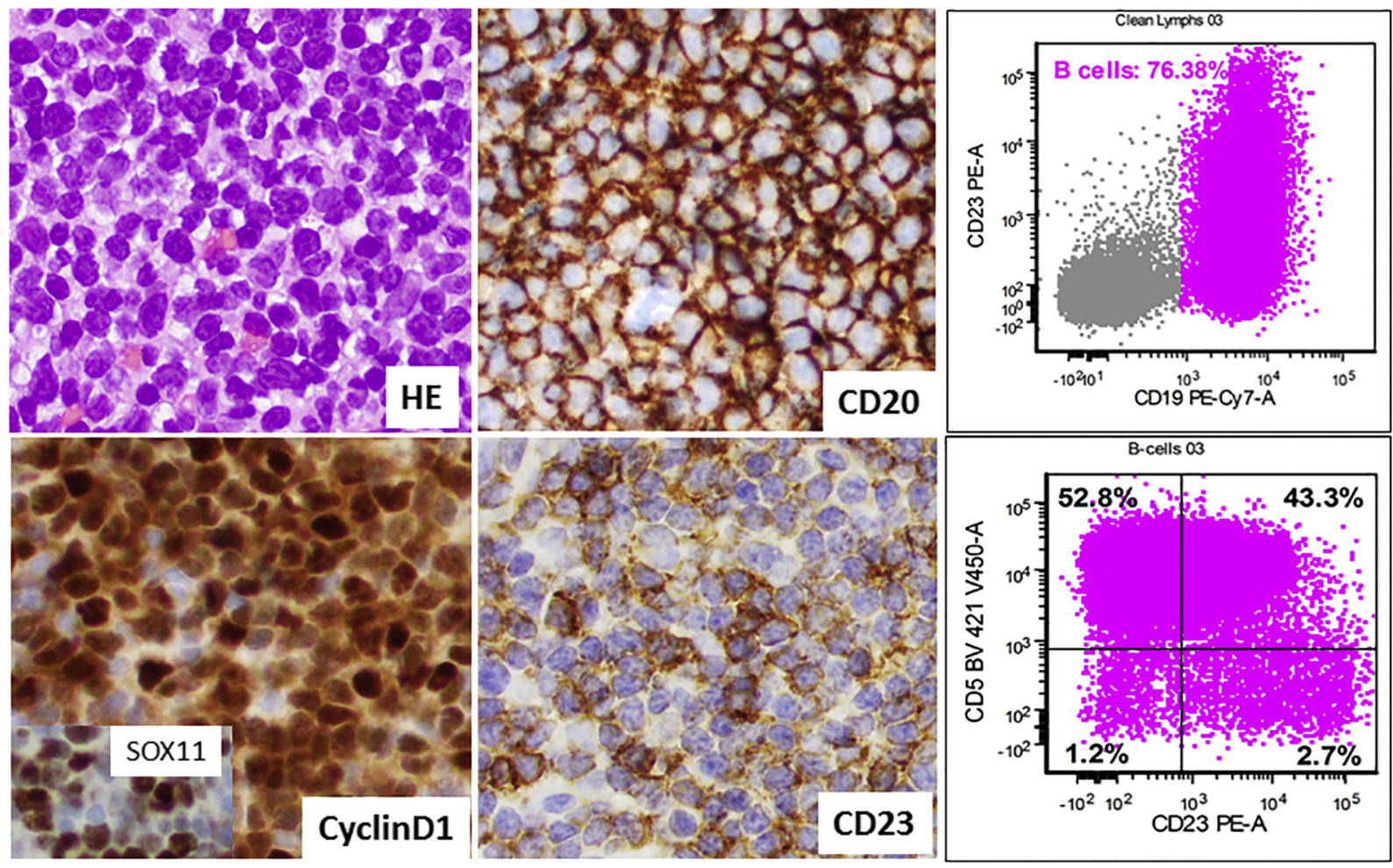

Figure 1 

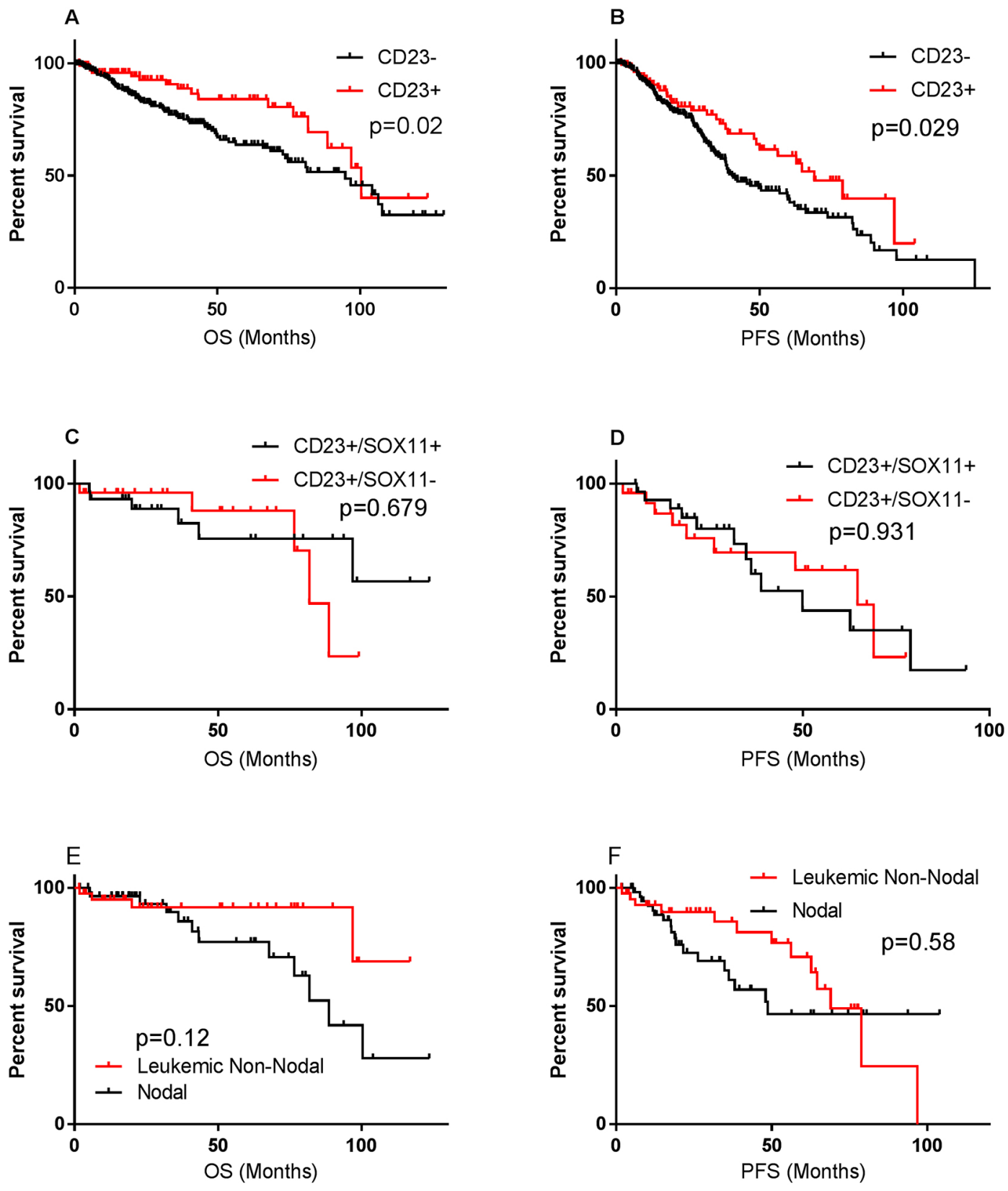

Figure 2 

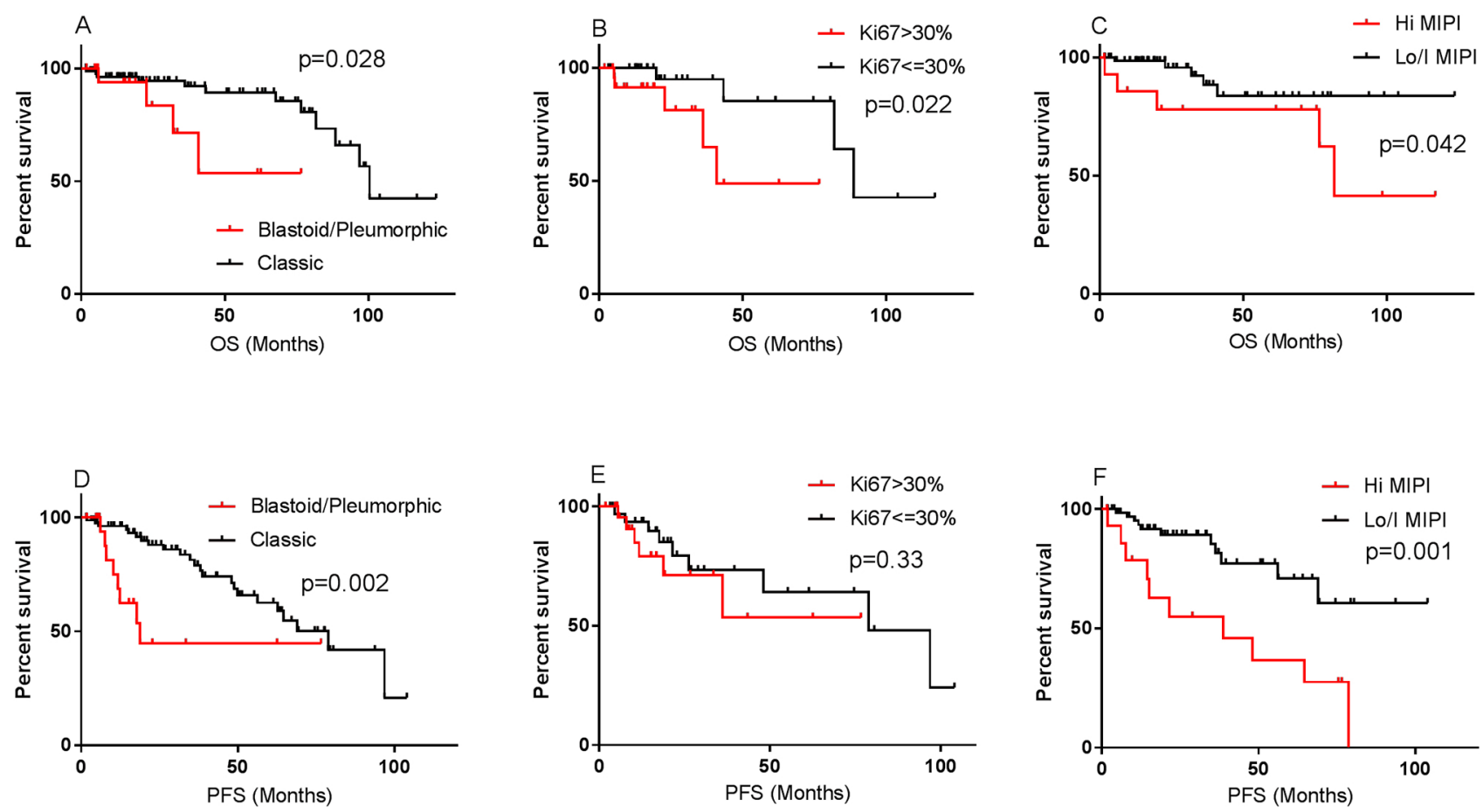

Figure 3 

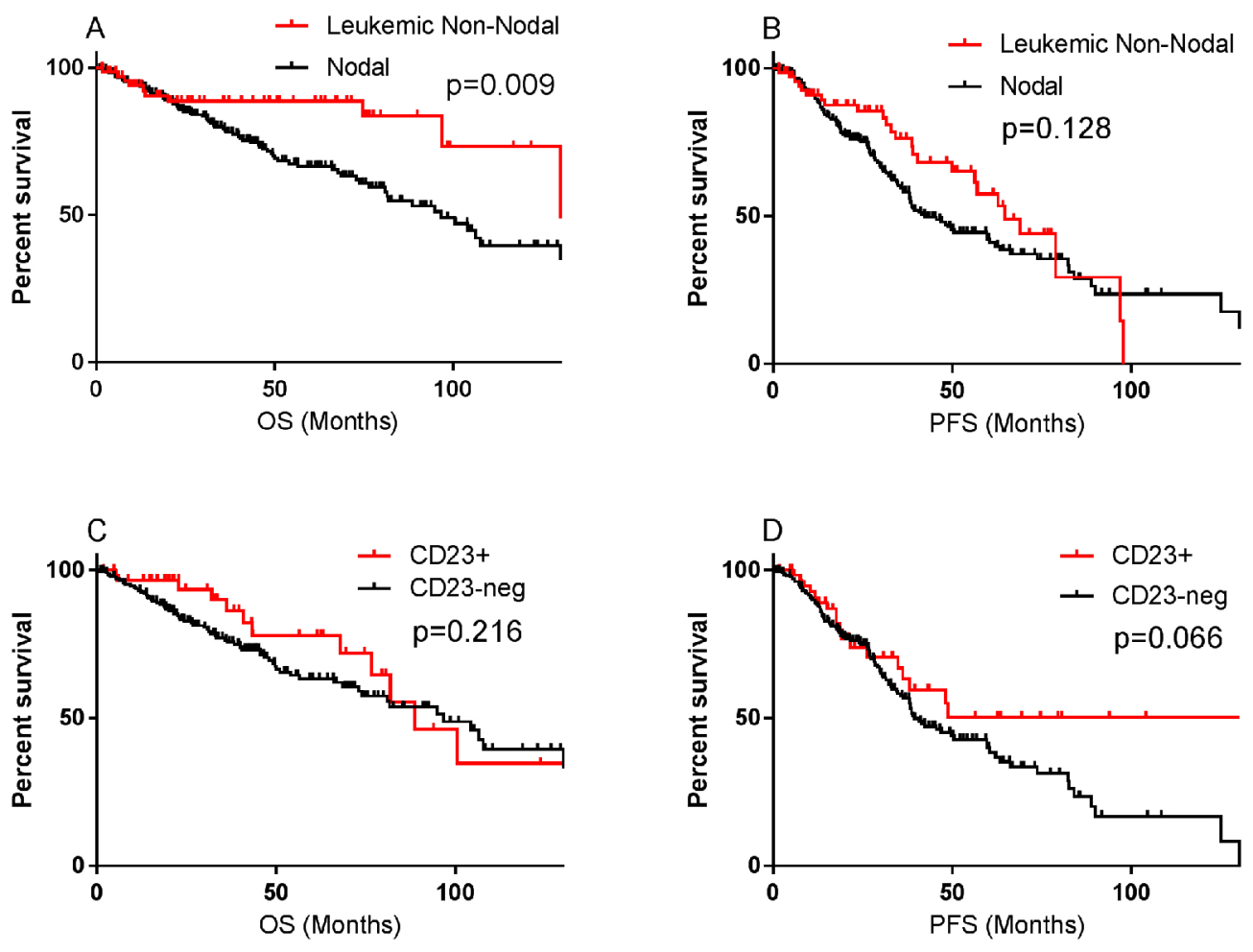

Figure 4 